\title{
Baixa prevalência de infecções por Clostridioides difficile em Hospitais de Referência em Oncologia
}

\author{
Low prevalence of Clostridioides difficile infection in Reference Hospitals in Oncology \\ Baja prevalencia de infecciones por Clostridioides difficile en Hospitales de Referencia Oncológica
}

Recebido: 29/04/2021 | Revisado: 05/05/2021 | Aceito: 10/05/2021 | Publicado: 26/05/2021

\author{
Catarina Tenório \\ ORCID: https://orcid.org/0000-0002-1119-0116 \\ Universidade Federal de Pernambuco, Brasil \\ E-mail: catarina_tl@yahoo.com.br \\ Paulo Sérgio Ramos de Araújo \\ ORCID: https://orcid.org/0000-0002-7839-0737 \\ Universidade Federal de Pernambuco, Brasil \\ E-mail: psergiora@gmail.com \\ Ana Kelly Lins \\ ORCID: https://orcid.org/0000-0002-1866-9664 \\ Laboratório Marcelo Magalhães, Pernambuco, Brasil \\ E-mail: akellylins@gmail.com \\ Carlos Roberto Weber Sobrinho \\ ORCID: https://orcid.org/0000-0002-2188-6061 \\ Universidade Federal de Pernambuco, Brasil \\ E-mail: carlos.sobrinho@ufpe.br \\ Vera Magalhães \\ ORCID: https://orcid.org/0000-0003-3495-0234 \\ Universidade Federal de Pernambuco, Brasil \\ E-mail: vemagalhaes@uol.com.br
}

\begin{abstract}
Resumo
As infecções por Clostridioides difficile (CDI) aumentaram em gravidade e incidência durante a última década. O câncer predispõe os pacientes à $\mathrm{CDI}$, devido à maior exposição aos fatores de risco. O presente estudo teve como objetivo determinar a prevalência, resposta clínica, desfecho, descrever os fatores de risco estabelecidos na literatura em pacientes oncológicos com CDI. O estudo foi realizado em dois serviços de referência em oncologia de Recife/PE e envolveu pessoas com idade $\geq 18$ anos, que apresentaram o quadro de diarreia após 48horas da admissão hospitalar, no período de novembro/2017 a agosto/2019. Para o diagnóstico de CDI foi utilizado o exame Reação em Cadeia da Polimerase em Tempo Real (qRT-PCR). Um total de 156 pacientes foram incluídos, a CDI foi identificada em 7,05\% (11/156) dos pacientes estudados, todos isolados foram rastreados e amplificados por qRT-PCR quanto à presença dos genes que codificam as toxinas A e B $(t c d A$ e $t c d B)$, toxina binária $(c d t A)$ e triose fosfato isomerase $(t p i)$. O câncer de mama $27,3 \%$ (3/11) e a leucemia linfóide aguda 18,2\% (2/11) foram os cânceres mais frequentes; 90,9\% (10/11) fizeram uso de antibióticos e 63,6\% (7/11) dos casos evoluíram para óbito. Apesar de utilizar a técnica qRT-PCR, que é a mais sensível e específica, a prevalência de CDI foi baixa 7,05\% (11/156), não houve registros de casos de CDI graves, sendo a maioria dos casos leves, o que sugere uma circulação de cepas com baixa virulência que determinam menor morbidade e mortalidade.
\end{abstract}

Palavras-chave: Clostridioides difficile infecção; Câncer; Antibióticos; Quimioterápicos; qRT-PCR.

\begin{abstract}
The severity and incidence of Clostridioides difficile infection (CDI) have increased over the last decade. Cancer predisposes patients to CDI due to increased exposure to risk factors. The present study aimed to determine the prevalence, clinical response, outcomes, risk factors as supported in the literature in cancer patients with CDI. This was a prospective cross-sectional study conducted at two reference centres in oncology in Recife-PE, Brazil and involved individuals aged $\geq 18$ years who presented with diarrhoea 48 hours after hospital admission, from November 2017 to August 2019. CDI was diagnosed using real-time polymerase chain reaction (qRT-PCR). A total of 156 patients were included in the study, and CDI was detected in $7.05 \%(11 / 156)$ of the patients. All isolates were screened, and the DNA was isolated and amplified by qRT-PCR for the detection of genes coding for toxins A and B (tcdA and $t c d B)$, binary toxin $(c d t A)$, and triose phosphate isomerase $(t p i)$. Breast cancer and acute lymphoid leukemia were the most frequent cancers $(27.3 \%$ [3/11] and $18.2 \%$ [2/11], respectively); 90.9\% (10/11) of the cases used antibiotics, and the mortality rate was $63.6 \%$ (7/11 patients). Despite the use of the qRT-PCR technique, which is the most sensitive and specific method for diagnosing CDI, it was found that the prevalence of the disease was low $(7.05 \% ; 11 / 156)$. There were no
\end{abstract}


cases of severe CDI, and most cases found were mild, which suggests the circulation of strains with low virulence that determine lower morbidity and mortality.

Keywords: Clostridioides difficile infection; Cancer; Antibiotics; Chemotherapy; qRT-PCR.

\section{Resumen}

Las infecciones por Clostridioides difficile (CDI) han aumentado en gravedad e incidencia durante la última década. El cáncer predispone a los pacientes a la CDI, debido a una mayor exposición a factores de riesgo. El presente estudio tuvo como objetivo determinar la prevalencia, respuesta clínica, resultado, describir los factores de riesgo establecidos en la literatura en pacientes oncológicos con CDI. El estudio se llevó a cabo en dos servicios de referencia oncológica de Recife/PE e involucró a personas $\geq 18$ años, que presentaron diarrea a las 48 horas del ingreso hospitalario, de noviembre/2017 a agosto/2019. Para el diagnóstico de CDI se utilizó el examen de Reacción en Cadena de Polimerasa en Tiempo Real (qRT-PCR). Se incluyeron un total de 156 pacientes, la CDI se identificó en el 7,05\% (11/156) de los pacientes estudiados, todos los aislamientos se rastrearon y amplificaron mediante qRT-PCR para detectar la presencia de los genes que codifican las toxinas A y B ( $t c d A$ y $t c d B)$, toxina binaria $(c d t A)$ y triosa fosfato isomerasa (tpi). El cáncer de mama el 27,3\% (3/11) y la leucemia linfoide aguda el 18,2\% (2/11) fueron los cánceres más frecuentes; el 90,9\% (10/11) utilizaba antibióticos y el 63,6\% (7/11) de los casos fallecieron. A pesar de utilizar la técnica qRT-PCR, que es la más sensible y específica, la prevalencia de CDI fue baja 7,05\% (11/156), no se registraron casos de CDI grave, siendo la mayoría de casos leves, lo que sugiere una circulación de cepas de baja virulencia que determinan una menor morbilidad y mortalidad.

Palabras clave: Infección por Clostridioides difficile; Cáncer; Antibióticos; Quimioterápicos; qRT-PCR.

\section{Introdução}

As infecções por Clostridioides difficile (CDI) aumentaram em gravidade e incidência durante a última década (Fuereder et al., 2016) e representam a causa mais importante de diarreia hospitalar associada ao uso de antibióticos, com uma crescente incidência de mais de 250 mil casos/ano nos Estados Unidos da América (EUA) (Mcglone et al., 2012). O câncer predispõe os pacientes à CDI, devido a maior exposição aos fatores de risco, como o uso frequente de antibióticos de largo espectro e agentes quimioterápicos (Delgado et al., 2017).

A doença tem um espectro clínico amplo, variando desde diarreia leve e aquosa à colite pseudomembranosa, colite fulminante e morte (Rupnik, Wilcox \& Gerding, 2009). Na população oncológica onde a diarreia é um efeito adverso comum dos regimes quimioterápicos, o diagnóstico pode ser um desafio e precisa de um alto grau de suspeita clínica. Neste grupo, a detecção e o tratamento precoces da CDI são cruciais e estão associados a redução da mortalidade (Khan et al., 2012).

No Brasil, a infecção causada pelo $C$. difficile em pacientes oncológicos é certamente subnotificada, uma vez que o diagnóstico não tem sido rotina nos hospitais. Apesar da importância mundial, há poucas pesquisas na região sudeste e nordeste, sendo este o primeiro estudo em Pernambuco sobre Clostridiodes difficile.

Diante da crescente importância da CDI e da carência de investigações sobre o tema no Brasil, resolveu-se realizar esse estudo cujos objetivos principais foram determinar a prevalência, resposta clínica, desfecho e fatores de risco dos pacientes com diarreia nosocomial por $C$. difficile em dois centros de referência em oncologia em Recife-PE.

\section{Metodologia}

Trata-se de um estudo prospectivo, quantitativo, corte transversal para determinação da prevalência, resposta clínica, desfecho, assim como, descrever os fatores de risco (idade > 65 anos, antibioticoterapia prévia, presença de outras comorbidades, uso de quimioterápicos, neutropenia febril, uso de protetores gástricos, cirurgia prévia gastrintestinal e tempo de internamento) estabelecidos na literatura, realizado em dois serviços de referência em oncologia de Recife/PE, Brasil.

O estudo envolveu pessoas com idade $\geq 18$ anos, que apresentaram o quadro de diarreia após 48horas da admissão hospitalar, no período de novembro/2017 a agosto/2019. Para o diagnóstico foi utilizado o exame Reação em Cadeia da Polimerase em Tempo Real (qRT-PCR). 
Todos os isolados foram rastreados e amplificados quanto à presença dos genes $t c d A, t c d B, c d t A$ e tpi, usando primers conhecidos como descrito por Knetsch et al., (2011) e Kilic et al., (2015). O projeto foi submetido à análise do Comitê de Ética do Centro de Ciências da Saúde da Universidade Federal de Pernambuco, da Sociedade Pernambucana de Combate ao Câncer e do Comitê de Ética do Hospital das Clínicas com autorização, CAAE 63059516.7.0000.5208.

Os dados foram analisados descritivamente através de frequências absolutas e percentuais para as variáveis categóricas e das medidas: média, desvio padrão e mediana da variável idade. Para avaliar diferença entre duas variáveis categóricas foi utilizado o teste Qui-quadrado de Pearson ou o teste Exato de Fisher, quando a condição para utilização do teste Qui-quadrado não foi verificada. A margem de erro utilizada nos testes estatísticos foi de 5\%. Os dados foram digitados na planilha EXCEL e o programa utilizado para obtenção dos cálculos estatísticos foi o IMB SPSS na versão 23.

\section{Resultados}

$\mathrm{Na}$ Tabela 1, ressalta-se que no grupo total, a maioria 59,6\% (93/156) era do sexo feminino, e apenas 29,5\% (46/156) tinha idade avançada (65 anos ou mais). Um pouco mais da metade, 53,2\% (83/156) apresentava comorbidades. Entre os pacientes com neutropenia 21,8\% (34/156), a maioria 58,8\% (20/34) evoluiu com duração de 1 a 7 dias e 81,6\% (124/152) utilizou ranitidina como protetor gástrico. Os tipos de câncer mais frequentes foram: mama 15,4\% (24/156), linfoma não Hodkgin $14,1 \%(22 / 156)$, colo de útero 10,9\% (17/156), colorretal 9,6\% (15/156), leucemia mieloide aguda (LMA) 9,6\% (15/156) e leucemia linfoide aguda (LLA) 5,8\% (9/156). Não foram registradas diferenças significativas entre os grupos para nenhuma das variáveis analisadas. 
Tabela 1 - Características clínico-epidemiológicas de pacientes oncológicos segundo os resultados de CDI.

\begin{tabular}{|c|c|c|c|c|c|c|c|}
\hline \multirow{3}{*}{ Variável } & \multicolumn{4}{|c|}{ Grupo } & \multirow{2}{*}{\multicolumn{2}{|c|}{ Grupo total }} & \multirow{3}{*}{ Valor p } \\
\hline & \multicolumn{2}{|c|}{ CDI positivo } & \multicolumn{2}{|c|}{ CDI negativo } & & & \\
\hline & $\mathbf{N}$ & $\%$ & $\mathbf{N}$ & $\%$ & $\mathbf{N}$ & $\%$ & \\
\hline Sexo & & & & & & & $\mathrm{p}^{(1)}=0,201$ \\
\hline Masculino & 2 & 18,2 & 61 & 42,1 & 63 & 40,4 & \\
\hline Feminino & 9 & 81,8 & 84 & 57,9 & 93 & 59,6 & \\
\hline Idade avançada & & & & & & & $\mathrm{p}^{(1)}=0,509$ \\
\hline Sim & 2 & 18,2 & 44 & 30,3 & 46 & 29,5 & \\
\hline Não & 9 & 81,8 & 101 & 69,7 & 110 & 70,5 & \\
\hline Comorbidades & & & & & & & $\mathrm{p}^{(2)}=0,016^{*}$ \\
\hline $\operatorname{Sim}$ & 2 & 18,2 & 81 & 55,9 & 83 & 53,2 & \\
\hline Não & 9 & 81,8 & 64 & 44,1 & 73 & 46,8 & \\
\hline Neutropenia (neutrófilos $\leq 500 / \mathbf{m m}^{3}$ ) & & & & & & & $\mathrm{p}^{(1)}=0,257$ \\
\hline $\operatorname{Sim}$ & 4 & 36,4 & 30 & 20,7 & 34 & 21,8 & \\
\hline Não & 7 & 63,6 & 115 & 79,3 & 122 & 78,2 & \\
\hline Uso de protetores gástricos & & & & & & & $\mathrm{p}^{(1)}=0,393$ \\
\hline Ranitidina & 7 & 70,0 & 117 & 82,4 & 124 & 81,6 & \\
\hline Omeprazol & 3 & 30,0 & 25 & 17,6 & 28 & 18,4 & \\
\hline Tempo internamento (antes coleta fezes) & & & & & & & $\mathrm{p}^{(1)}=0,168$ \\
\hline Até 7 dias & 3 & 27,3 & 67 & 46,2 & 70 & 44,9 & \\
\hline De $8-14$ dias & 2 & 18,2 & 41 & 28,3 & 43 & 27,6 & \\
\hline Mais de14 dias & 6 & 54,5 & 37 & 25,5 & 43 & 27,6 & \\
\hline Tipo de Câncer & & & & & & & $\mathrm{p}^{(1)}=0,385$ \\
\hline Colo de útero & 2 & 18,2 & 15 & 10,3 & 17 & 10,9 & \\
\hline Bexiga & 1 & 9,1 & - & - & 1 & 0,6 & \\
\hline Colorretal & - & - & 15 & 10,3 & 15 & 9,6 & \\
\hline Gástrico & 1 & 9,1 & 8 & 5,5 & 9 & 5,8 & \\
\hline Leiomiossarcoma uterino & 1 & 9,1 & - & - & 1 & 0,6 & \\
\hline Leucemia linfoide aguda (LLA) & 2 & 18,2 & 7 & 4,8 & 9 & 5,8 & \\
\hline Leucemia mieloide aguda (LMA) & 1 & 9,1 & 14 & 9,7 & 15 & 9,6 & \\
\hline Linfoma não Hodkgin & - & - & 22 & 15,2 & 22 & 14,1 & \\
\hline Mama & 3 & 27,3 & 21 & 14,5 & 24 & 15,4 & \\
\hline
\end{tabular}

Abreviações: CDI, Infecção por Clostridioides difficile. Fonte: Dados da pesquisa (2020).

(*) Diferença significativa a 5\%;

(1) Através do teste Exato de Fisher.

Fonte: Autores.

A Tabela 2 mostra que: no grupo total a maioria 69.2\% (108/156) fez uso de antibiótico prévio (até 4 semanas antes da data da coleta das fezes). Entre os antibióticos mais utilizados foram: piperacilina + tazobactam 40,7\% (44/108), meropenem 28,7\% (31/108) e cefepima 25,9\% (28/108). Piperacilina + tazobactam foi o único medicamento com diferença significativa (p $<0,05)$ entre os grupos. A maioria 62,2\% (97/156) realizou quimioterapia e os quimioterápicos mais frequentes foram: agentes antimetabólitos 52,6\% (51/97), antraciclinas (doxorrubicina, daunorrubicina, idarrubicina) 42,3\% (41/97) e agentes platinantes $33,0 \%$ (32/97). Não foram registradas diferenças significativas $(\mathrm{p}<0,05)$ entre os grupos para nenhuma das variáveis. 
Tabela 2 - Principais antibióticos e quimioterápicos utilizados em pacientes oncológicos segundo os resultados de CDI.

\begin{tabular}{|c|c|c|c|c|c|c|c|}
\hline \multirow{3}{*}{ Potenciais fatores de risco } & \multicolumn{4}{|c|}{ Grupo } & \multirow{2}{*}{\multicolumn{2}{|c|}{ Grupo total }} & \multirow{3}{*}{ Valor p } \\
\hline & \multicolumn{2}{|c|}{ CDI positivo } & \multicolumn{2}{|c|}{ CDI negativo } & & & \\
\hline & $\mathbf{N}$ & $\%$ & $\mathbf{N}$ & $\%$ & $\mathbf{N}$ & $\%$ & \\
\hline Antibicoterapia prévia (4 semanas antes coleta da amostra) & & & & & & & $\mathrm{p}^{(1)}=0,174$ \\
\hline Sim & 10 & 90,9 & 98 & 67,6 & 108 & 69,2 & \\
\hline Não & 1 & 9,1 & 47 & 32,4 & 48 & 30,8 & \\
\hline \multicolumn{8}{|l|}{ Antibióticos utilizados previamente } \\
\hline Cefepima & & & & & & & $\mathrm{p}^{(1)}=1,000$ \\
\hline Sim & 2 & 20,0 & 26 & 26,5 & 28 & 25,9 & \\
\hline Não & 8 & 80,0 & 72 & 73,5 & 80 & 74,1 & \\
\hline Piperacilina + Tazobactam & & & & & & & $\mathrm{p}^{(1)}=0,046^{*}$ \\
\hline Sim & 1 & 10,0 & 43 & 43,9 & 44 & 40,7 & \\
\hline Não & 9 & 90,0 & 55 & 56,1 & 64 & 59,3 & \\
\hline Meropenem & & & & & & & $\mathrm{p}^{(1)}=0,721$ \\
\hline $\operatorname{Sim}$ & 2 & 20,0 & 29 & 29,6 & 31 & 28,7 & \\
\hline Não & 8 & 80,0 & 69 & 70,4 & 77 & 71,3 & \\
\hline Quimioterapia (30 dias antes do episódio diarreico) & & & & & & & $\mathrm{p}^{(1)}=1,000$ \\
\hline $\operatorname{Sim}$ & 7 & 63,6 & 90 & 62,1 & 97 & 62,2 & \\
\hline Não & 4 & 36,4 & 55 & 37,9 & 59 & 37,8 & \\
\hline \multicolumn{8}{|l|}{ Classe dos quimioterápicos } \\
\hline Agentes antimetabólitos & & & & & & & $\mathrm{p}^{(1)}=0,705$ \\
\hline $\operatorname{Sim}$ & 3 & 42,9 & 48 & 53,3 & 51 & 52,6 & \\
\hline Não & 4 & 57,1 & 42 & 46.7 & 46 & 47,4 & \\
\hline Antraciclinas & & & & & & & $\mathrm{p}^{(1)}=0,451$ \\
\hline Sim & 4 & 57,1 & 37 & 41,1 & 41 & 42,3 & \\
\hline Não & 3 & 42,9 & 53 & 58,9 & 56 & 57,7 & \\
\hline Agentes platinantes & & & & & & & $\mathrm{p}^{(1)}=0,420$ \\
\hline Sim & 1 & 14,3 & 31 & 34,4 & 32 & 33,0 & \\
\hline Não & 6 & 85.7 & 59 & 65,6 & 65 & 67,0 & \\
\hline
\end{tabular}

Abreviações: CDI, Infecção por Clostridioides difficile. Fonte: Dados da pesquisa (2020).

(*) Diferença significativa a 5\%

(1) Através do teste Exato de Fisher

Fonte: Autores.

No grupo CDI positivo, 18,2\% (2/11) foram tratados para diarreia hospitalar, enquanto que no grupo CDI negativo, esse percentual foi de 13,1\% (19/145). Entre os pacientes que fizeram uso da medicação, 5,8\% (9/21) foi tratado para CDI com metronidazol VO e 8,3\% (13/21) com metronidazol EV. Dos 21 tratados, 81,0\% (17/21) evoluiu com resolução dos sintomas e $19,0 \%(4 / 21)$ com resposta parcial. No grupo total, o desfecho foi de cura para 60,3\% (94/156) dos pacientes e óbito para 39,7\% (62/156), conforme resultados apresentados na Tabela 3. Não foram registradas diferenças significativas $(p<0,05)$ entre os grupos. 
Tabela 3 - Tratamento para diarreia hospitalar e desfecho dos casos segundo os resultados de CDI.

\begin{tabular}{|c|c|c|c|c|c|c|c|}
\hline \multirow{3}{*}{ Variável } & \multicolumn{4}{|c|}{ Grupo } & & & \multirow{3}{*}{ Valor $\mathbf{p}$} \\
\hline & \multicolumn{2}{|c|}{ CDI positivo } & \multicolumn{2}{|c|}{ CDI negativo } & \multicolumn{2}{|c|}{ Grupo total } & \\
\hline & $\mathbf{n}$ & $\%$ & $\mathbf{N}$ & $\%$ & $\mathbf{N}$ & $\%$ & \\
\hline Tratamento para C. Difficile & & & & & & & $\mathrm{p}^{(1)}=0,644$ \\
\hline $\operatorname{Sim}$ & 2 & 18,2 & 19 & 13,1 & 21 & 13,5 & \\
\hline Não & 9 & 81,8 & 126 & 86,9 & 135 & 86,5 & \\
\hline Metronidazol VO & & & & & & & $\mathrm{p}^{(1)}=1,000$ \\
\hline Sim & - & - & 9 & 6,2 & 9 & 5,8 & \\
\hline Não & 11 & 100,0 & 136 & 93,8 & 147 & 94,2 & \\
\hline Metronidazol EV & & & & & & & $\mathrm{p}^{(1)}=0,229$ \\
\hline $\operatorname{Sim}$ & 2 & 18,2 & 11 & 7,6 & 13 & 8,3 & \\
\hline Não & 9 & 81,8 & 134 & 92,4 & 143 & 91,7 & \\
\hline Resposta clínica dos casos tratados com antibióticos & & & & & & & $\mathrm{p}^{(1)}=1,000$ \\
\hline Resolução sintomas & 2 & 100,0 & 15 & 78,9 & 17 & 81,0 & \\
\hline Resposta parcial & - & - & 4 & 21,1 & 4 & 19,0 & \\
\hline Desfecho & & & & & & & $\mathrm{p}^{(1)}=0,115$ \\
\hline Cura & 4 & 36,4 & 90 & 62,1 & 94 & 60,3 & \\
\hline Óbito & 7 & 63,6 & 55 & 37,9 & 62 & 39,7 & \\
\hline
\end{tabular}

Abreviações: CDI, Infecção por Clostridioides difficile. Fonte: Dados da pesquisa (2020).

(1) Através do teste Exato de Fisher

Fonte: Autores.

\section{Discussão}

Apesar dos esforços para prevenir a CDI nos últimos anos, sua incidência vem aumentando no mundo (Raza et al., 2010), com uma variabilidade nas diferentes regiões, ressaltando a importância das investigações regionais. No leste da China, Fang et al., (2014) encontraram uma prevalência de 20,5\% de CDI em três hospitais oncológicos; na Espanha esse percentual foi de 17,3\% (Garzotto et al, 2015) e nos EUA, a incidência de CDI foi de aproximadamente 7-14\% em adultos com câncer (Scappaticci et al., 2017).

No Brasil, há particularidades em relação à prevalência de CDI nas diferentes regiões. Pires et al., (2014), Balassiano et al., (2010) e Cecília Costa (2014) detectaram uma incidência de CDI que variou de 8,3\% até 46,3\%, percentuais maiores que o obtido no presente estudo (7,05\%). Mesmo utilizando a qRT-PCR, que é o método mais sensível e específico, nossos resultados apresentaram menor prevalência de CDI que as demais pesquisas brasileiras uma vez que o ELISA, técnica utilizada pela maioria, pode gerar resultados falso-positivos.

Ademais, os pacientes identificados com CDI foram predominantemente mulheres jovens, o que está relacionado com a elevada incidência de câncer de colo de útero no Brasil (INCA, 2019). PÉPIN et al., (2005), demonstraram que o risco de contrair a infecção durante um surto foi 10 vezes maior entre pessoas com mais de 65 anos de idade, pois a microbiota intestinal diminui em número com o envelhecimento (Lessa, Gould \& McDonald, 2012; Krishna et al., 2013). Além disso, nossa baixa prevalência pode estar associada à maior inclusão de pacientes jovens, diferentemente dos países desenvolvidos, onde a população alvo é composta por mais idosos.

Por outro lado, a influência da supressão ácida no estômago permanece incerta na CDI, mesmo com a viabilidade dos esporos do C.difficile no Ph gástrico (Leffler \& Lamont, 2009). Alguns investigadores relataram um risco aumentado de infecção associado à supressão ácida (Dial et al., 2004) enquanto outros, depois de se ajustarem à coexistência das condições, não confirmaram um aumento do risco (Kyne et al., 2002; Novack et al., 2014). Entre os casos positivos, muitos não apresentaram 
neutropenia, ainda que a mesma esteja associada a altas taxas de CDI nos pacientes oncológicos (Chul, Ki-Eun \& Hak-Ryul, 2016; Delgado et al, 2017).

$\mathrm{Na}$ literatura, o uso prévio de agentes antimicrobianos como clindamicina, fluoroquinolonas, cefalosporinas, monobactâmicos e carbapenêmicos resulta em uma disbiose que favorece o crescimento para o C. Difficile (Gorschüter et al., 2001), constituindo o principal fator de risco para CDI (Brown et al., 2013; Britton \& Young, 2014). Além disso, o antimicrobiano mais utilizado foi a piperacilina+tazobactam, com uma diferença estatisticamente significante entre os dois grupos. Resultados semelhantes foram descritos por Chang et al., (2016) nos EUA.

Embora o câncer hematológico apresente um risco de infecção por $C$. difficile de 2,5 vezes quando comparado ao TU sólido, fato que é atribuído a uma permanência prolongada durante as hospitalizações, não encontramos uma variação nas taxas de CDI com base no tipo de câncer (Hebbard et al., 2016; Abughanemeh et al., 2018).

Geralmente o uso dos antibióticos está relacionado ao desenvolvimento de CDI. Contudo, a quimioterapia tem sido descrita como um fator de risco para o desenvolvimento de CDI (Khan et al., 2012). Vários agentes quimioterápicos estão associados ao desenvolvimento de CDI (Chopra, Alangaden \& Chandrasckar, 2010). Por outro lado, Fuereder et al., (2016), avaliaram que a quimioterapia não foi um fator de risco significativo para CDI, em comparação com a exposição prévia aos antibióticos.

\section{Considerações Finais}

Diante da importância mundial do $C$. difficile, o presente trabalho contribuiu com o conhecimento dos dados epidemiológicos da CDI em pacientes imunocomprometidos no estado de Pernambuco. Os resultados encontrados auxiliaram a compreensão dos mecanismos envolvidos no processo de aquisição da CDI no ambiente hospitalar, além de promover estratégias de prevenção para evitar a disseminação do microrganismo nessa população de risco.

Em conclusão, é possível que a baixa virulência das cepas tenha tido um papel preponderante na baixa prevalência visto que a maioria dos casos positivos evoluiu com quadro clínico leve a moderado, sem registro de recidivas no seguimento de 3060 dias. A mortalidade desses pacientes pode ser explicada pela gravidade do tipo de câncer, pois nenhum paciente apresentou colite pseudomembranosa grave que justificasse os óbitos. Esses dados reforçam que mais estudos de prevalência são fundamentais para a vigilância de casos de CDI nas unidades hospitalares brasileiras.

\section{Referências}

Abughanimeh, O., Qasrawi, A., Kaddourah, O., Momani, L. A. \&, Ghanimeh, M. A. (2018). Clostridium difficile infection in oncology patients: epidemiology, pathophysiology, risk factors, diagnosis, and treatment. Hospital Practice, 46(5), 266-277. https://doi.org/10.1080/21548331.2018.1533673.

Balassiano, I. T., Santos-Filho, J., Oliveira, M. P. B., Ramos, M. C., Japiassu, A. M., Reis, A. M., Brazier, J. S., Ferreira, E. O., \& Domingues, R. M. C. P. (2010). An outbreak case of Clostridium difficile-associated diarrhea among elderly inpatients of an intensive care unit of a tertiary hospital in Rio de Janeiro, Brazil. Diagnostic Microbiology and Infectious Diseases, 68(4), 449-55.

Britton, R. A., \& Young, V. B. (2014). Role of the intestinal microbiota in resistance to colonization by Clostridium difficile. Gastroenterology, 146, 1547-53.

Brown, K. A., Khanafer, N., Daneman, N., \& Fismana, D.N. (2013). Meta-analysis of antibiotics and the risk of community-associated Clostridium difficile infection. Antimicrobial Agents and Chemotherapy, 57(5), 2326-32.

Chang, G. Y., Dembry, L. M., \& Banach, D. B. (2016). Epidemiology of Clostridium difficile infection in hospitalized oncology patients. American Journal of Infection Control, 44(11), 1408-1410.

Chopra,. T, Alangaden, G. J., \& Chandrasekar, P. (2010). Clostridium difficile infection in Cancer patients and Hematopoietic Stem Cell Transplant Recipients. Expert Review of Anti-infective Therapy, 8, 1113-1119.

Chul, P., Ki-Eun, H., \& Hak-Ryul, K. (2016). Overview of Clostridium difficile Infection in Cancer Patients. Journal of Infectious Diseases \& Diagnosis, 1, 109.

Costa, C. L., Carvalho, C. B. M., González, R. H., Gifoni, M. A. C., Ribeiro, R. A., Quesada-Gómez, C., \& Brito, G. A. C. (2017). Molecular epidemiology of Clostridium difficile infection in a Brazilian cancer hospital. Anaerobe, 48, 232-36. https://doi.org/10.1016/j.anaerobe.2017.10.001. 
Delgado, A., Reveles, I. A., Cabello, F. T., \& Reveles, K. R. (2017). Poorer outcomes among cancer patients diagnosed with Clostridium difficile infections in United States community hospitals. BMC Infectious Diseases, 17, 448. https://doi.org/10.1186/s12879-017-2553-z.

Dial, S., Alrasadi, K., Manoukian, C., Huang, A., \& Menzies, D. (2004). Risk of Clostridium difficile diarrhea among hospital inpatients prescribed proton pump inhibitors: cohort and case-control studies. CMAJ, 171(1), 33-38.

Fang, W. J., Da-Zhi, J., Yun, L., Cai-Yun, F., Peng, Z., Jiong, Q., Bing-Ru, T., Xiao-Gang, C., Yu-Long, Z., Jing, D., Wei-Hua, Z., Xue-Ren, F., Fan-Long, L., Xiao-Zhou, M., \& Shu-Sen, Z. (2014). Clostridium difficile carriage in hospitalized cancer patients: a prospective investigation in eastern China. BMC Infectious Diseases, 14:523. https://doi.org/10.1186/1471-2334-14-523.

Fuereder, T., Koni, D., Gleiss, A., Kund, M., Makristathis, A., Zielinski, C., \& Steininger, C. (2016). Risk factors for Clostridium difficile infection in hematooncological patients: A case control study in 144 patients. Scientific Reports, 6, 31498.

Garzotto, A. R., García, A. M., Unceta, N. M., Lopez, M. M., Orellana-Miguel, M. A., Díaz-García, C. V., Cortijo-Cascajares, S., Cortes-Funes, H., \& AgullóOrtuño, M. T. (2015). Risk factors associated with Clostridium difficile infection in adult oncology patients. Support Care Cancer, 23 , $1569-1577$. https://doi.org/ 10.1007/s00520-014-2506-7.

Gorschlüter, M., Glasmacher, A., Hahn, C., Schakowski, F., Ziske, C., Molitor, E., Marklein, G., Sauerbruch, T., \& Schmidt-Wolf, I. G. (2001). Clostridium difficile infection in patients with neutropenia. Clinical Infectious Diseases, 33, 786-791.

Hebbard, A. I., Slavin, M. A., Reed, C., Trubiano, J. A., The, B. W., Haeusler, G. M., Thursky, K. A, \& Worth, L. J. (2016). The epidemiology of Clostridium difficile infection in patients with cancer. Expert Review of Anti-infective Therapy, 14, 1077-1085.

Khan, A., Raza, S., Batul, S. A., Khan, M., Aksoy, T., Baig, M. A., \& Berger, B. J. (2012). The evolution of Clostridium difficile infection in cancer patients: epidemiology, pathophysiology, and guidelines for prevention and management. Recent Patents Antiinfective Drug Discovery, 7(2), 157-70.

Kilic, A., Alam, M. J., Tisdel, N. L., Shan, D. N., Yapar, M., Yapar, M., Lasco, T. M., \& Garey, K. W. (2015). Multiplex Real-Time PCR Method for Simultaneous Identification and Toxigenic Type Characterization of Clostridium difficile From Stool Samples. Annals of laboratory medicine, 35:306-313.

Knetsch, C. W., Bakker, D., De Boer, R. F, Sanders, I., Hofs, S., Kooistra-Smid, A. M. D, Corver, J., Eastwood, K., Wilcox, M. H., \& Kuijper, E. J. (2011). Comparison of Real-Time PCR Techniques to Cytotoxigenic Culture Methods for Diagnosing Clostridium difficile Infection. Journal of clinical microbiology, 49(1), 227-231.

Krishna, S. G., Zhao, W., Apewokin, S. K., Krishna, K., Chepyala, P., \& Anaissie. E. J. (2013). Risk factors, preemptive therapy, and antiperistaltic agents for Clostridium difficile infection in cancer patients. Transplant Infectious Diseases, 15, 493-501.

Kyne, L., Sougioultzis, S., McFarland, L. V., \& Kelly, C. P. (2002). Underlying disease severity as a major risk factor for nosocomial Clostridium difficile diarrhea. Infection Control \& Hospital Epidemiology, 23, 653-9.

Leffler, D. A., \& Lamont, J. T. (2009). Treatment of Clostridium difficile-associated disease. Gastroenterology, $136,1899-912$.

Lessa, F. C., Gould, C. V., \& Mcdonald, L. C. (2012). Current status of Clostridium difficile infection epidemiology. Clinical infectious diseases, 55(2), 65-70.

Mcglone, S. M., Bailey, R. R., Zimmer, S. M., Popovich, M. J., Tian, Y., Ufberg, P., Muder, R. R., \& Lee, B. Y. (2012). The economic burden of Clostridium difficile. Clinical Microbiology and Infection, 18(3), 282-289.

MS/INCA/Estimativa de Câncer no Brasil, 2020- disponível em https://www.inca.gov.br/numeros-de-cancer.

Novack, L., Kogan, S., Gimpelevich, L., Howell, M., Borer, A., Kelly, C. P., Leffler, D. A., \& Novack, V. (2014). Acid suppression therapy does not predispose to Clostridium difficile infection: the case of the potential bias. PLoS One, 9(10), e110790.

Pépin, J., Valiquette, L., \& Cossette, B. (2005). Mortality attributable to nosocomial Clostridium difficile-associated disease during na epidemic caused by a hypervirulent strain in Quebec. CMAJ, 173, 1037-42.

Pereira, A. S., Shitsuka, D. M., Parreira, F. J., \& Shitsuka, R. (2018). Metodologia da pesquisa científica. UFSM.

Pires, R. N., Monteiro, A. A., Carneiro, L. C., Baethgen, L. F., Tavares, R., Lincho, C. S., Park, S., Perlin, D., Rodrigues, F. E. M., \& Pasqualotto, A. C. (2014). Clostridium difficile infection in Brazil: a neglected problem? American Journal of Infection Control, 42(4), 459-60.

Raza, S., Baig, M. A., Russell, H., Gourdet, Y., \& Berger, B. J. (2010). Clostridium difficile infection following chemotherapy. Recent Patents Antiinfective Drug Discovery, 5(1), 1-9.

Rupnik, M., Wilcox, M. H., \& Gerding, D. N. (2009). Clostridium difficile infection: new developments in epidemiology and pathogenesis. Nature Reviews Microbiology, 7(7), 526-36.

Scappaticci, G. B., Perissinotti, A. J., Nagel, J. L., Bixby, D. L., \& Marini, B. L. (2017). Risk factors and impact of Clostridium difficile recurrence on haematology patients. Journal of Antimicrobial Chemotherapy, 72(5), 1488-1495. 